\title{
Advances in High Intensity e-Beam Diode Development for Flash X-Ray Radiography
}

\author{
B.V. Oliver*, K. Hahn, M.D. Johnston and S. Portillo \\ Sandia National Laboratories, Albuquerque, New Mexico, USA
}

\begin{abstract}
Recent experiments at Sandia National Laboratories have demonstrated an electron beam diode X-ray source capable of producing $>350 \mathrm{rad}$ at one meter with $1.7 \mathrm{~mm}$ FWHM X-ray source distribution, with a $50 \mathrm{~ns}$ pulse-width and X-ray photon endpoint energy spectrum in the 6-7 MeV range. The diode operates at current densities of $\approx 1 \mathrm{MA} / \mathrm{cm}^{2}$. The intense electron beam rapidly $(\approx 5 \mathrm{~ns})$ heats the X-ray conversion anode/target, liberating material in the form of low density ion emission early in the pulse and high density plasma later. This environment gives rise to beam/plasma collective effects which dominate the diode and beam characteristics, affecting the radiation properties (dose and spot-size). A review of the diode operation, the measured source characteristics and the simulation methods and diagnostics used to guide its optimization is given.
\end{abstract}

PACS numbers: 41.75.Fr, 41.75.Ht, 41.50.+h, 52.59.Mv, 87.59.B-

\section{Introduction}

A variety of electron beam diodes are being used and developed for the purpose of creating high-brightness, flash X-ray radiography sources. In these diodes, high energy (multi MeV), high current (multi kA), small spot (multi $\mathrm{mm}$ ) electron beams are generated and stopped in high atomic number anode-targets (typically Ta or W), producing bremsstrahlung radiation. Experimental results presented here are obtained on the Radiographic Integrated Test Stand (RITS) accelerator [1] which is an Induction Voltage Adder (IVA) developed at Sandia National Laboratories. In its present configuration, RITS-6 (see Fig. 1a), a Marx generator drives 6 parallel, $8 \Omega$ water dielectric pulse forming lines which in turn drive 6 induction adder cavities. The individual induction cells are capable of holding off $1.75 \mathrm{MV}$ each and are joined in series by a vacuum coaxial Magnetically Insulated Transmission Line (MITL) which delivers power from the cells to the diode region. The electron beam is generated on the cathode of the diode, accelerated in an anodecathode $(\mathrm{A}-\mathrm{K})$ vacuum gap and transported to a high atomic number converter target. RITS-6 is capable of delivering greater than $10 \mathrm{MV}, 75 \mathrm{kA}, 70 \mathrm{~ns}$ pulses to high impedance $(\geq 150 \Omega)$ electron beam diodes. Flexibility in the architecture via changes to the MITL geometry also allows us for the ability to deliver $\approx 7.5 \mathrm{MV}$, $150 \mathrm{kA}, 70 \mathrm{~ns}$ pulses to lower impedance $(\approx 50 \Omega)$ diodes.

The figure of merit (FOM) for the diode radiographic utility is based on the resolving power of the source and is crudely quantified by the photon intensity, defined as:

* corresponding author; e-mail: bvolive@sandia.gov
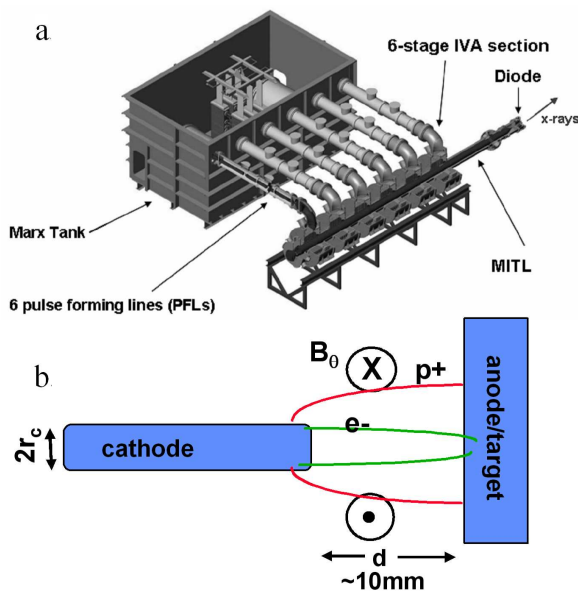

Fig. 1. (a) Illustration of RITS-6 Accelerator. (b) Illustration of the self-pinch diode.

$$
\mathrm{FOM}=\operatorname{dose} / \mathrm{spot}^{2}\left[\mathrm{rads} / \mathrm{mm}^{2}\right],
$$

where the radiation dose is defined at one meter. Recent experiments with the self-magnetic pinch (SMP) diode have demonstrated a radiation output $>350 \mathrm{rad}$ with a $1.7 \mathrm{~mm}$ FWHM X-ray source distribution, producing an $\mathrm{FOM} \approx 50 \mathrm{rad} / \mathrm{mm}^{2}$.

\section{The self-pinch diode}

The self-magnetic pinch (SMP) [2, 3] is a bipolar space-charge limited diode consisting of an anode and hollow cathode separated by a small A-K gap typically on the order of $1 \mathrm{~cm}$ (see Fig. 1b). Early in the pulse, electrons emitted from the cathode impinge the anode at a diameter that is larger than the cathode diameter with beam 
current densities $J>120 \mathrm{kA} / \mathrm{cm}^{2}$ and the electron beam heats the anode foil to $>400^{\circ} \mathrm{C}$ in a few nanoseconds, enabling space charge limited ion emission from the anode surface. The ion space charge provides nearly complete charge neutralization of the electron beam resulting in both an increased diode current and a pinching of the electron beam due to the self-magnetic field. The diode is conjectured to be a critically current limited system [2] such that the operating current can be described by

$$
I_{\mathrm{smp}}=8.5 \alpha \frac{r_{\mathrm{c}}}{d} \sqrt{\gamma^{2}-1} \quad[\mathrm{kA}]
$$

where $\alpha$ is an empirical scaling factor $\approx 2$ and is associated with the increased current due to the presence of ion space-charge $[2,4,5]$ and is dependent on geometry and the beam and ion space-charge profile (assuming a radially uniform space-charge limited distribution of ions results in $\alpha \approx 1.6[5])$. A typical diode current and voltage profile is given in Fig. 2a for SMP operation on the RITS-6 accelerator at $6.5 \mathrm{MeV}(\gamma=13.8)$ and geometric aspect ratio $r_{\mathrm{c}} / d=1 / 2$ (RITS shot 337 ). Also plotted is the diode impedance and dose rate. Early in time the diode operates with an impedance of $\approx 45 \Omega$, which decreases throughout the later half of the pulse. According to Eq. (2), for $\gamma \gg 1$, the impedance is approximately $Z=V / I_{\text {smp }} \approx(551 / 8.5)\left(d / r_{\mathrm{c}}\right)(1 / \alpha)$ suggesting that $\alpha \approx 2.8$. This factor is larger than that found for large aspect ratio pinches where $r_{\mathrm{c}} / d \gg 1$ and $\alpha \approx 1.6$ and even rod-pinches where $2.0<\alpha<2.6$ [5].

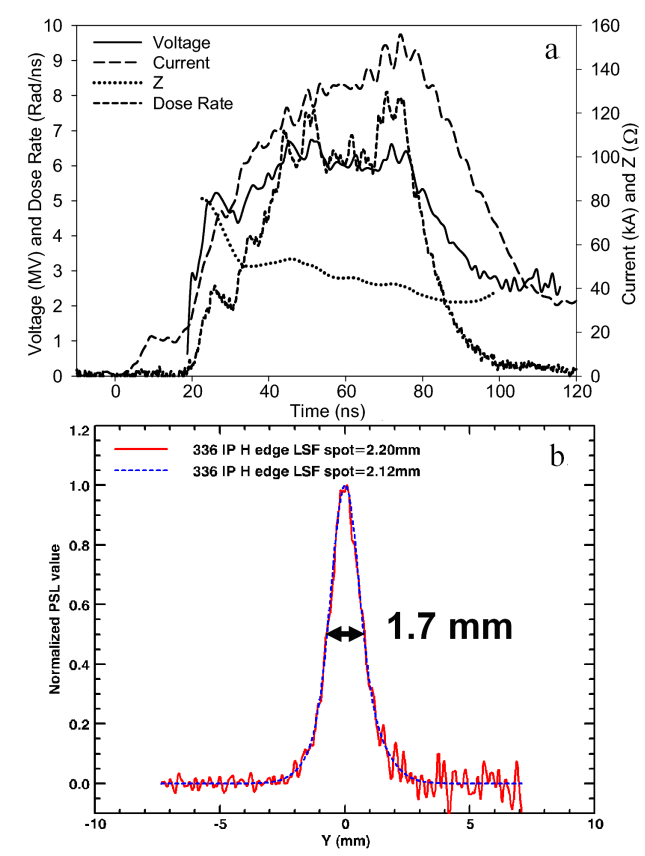

Fig. 2. (a) Typical operating characteristics for the SMP diode at $6.5 \mathrm{MeV}$ : voltage (solid), current (dash), impedance (dot), and dose rate (thick dash). (b) The line-spread-function for an SMP diode at $6.5 \mathrm{MeV}$, producing 350 rad@m (dot), also plotted is a double Gaussian fit (dash) which is characterized by a $1.7 \mathrm{~mm}$ FWHM.
We have suggested that the diode acts similar to a $1 / 4$ betatron focusing element operating in vacuum with the beam completely charge neutralized but current unneutralized. The focal length $F_{\text {smp }}$ and minimum beam diameter $a_{\mathrm{min}}$ on target can be approximated by

$$
F_{\mathrm{smp}} \approx \frac{r_{\mathrm{c}}}{2}\left(\frac{\pi I_{\mathrm{A}}}{I_{\mathrm{smp}}}\right)^{1 / 2}, \quad a_{\mathrm{min}} \approx \frac{2}{\sqrt{\pi}} \frac{\varepsilon}{\gamma \beta r_{\mathrm{c}}} F_{\mathrm{smp}},
$$

where $I_{\mathrm{A}}=17 \gamma \beta[\mathrm{kA}]$ is the Alfvén current, $\varepsilon[\mathrm{rad} / \mathrm{cm}]$ is the normalized root mean square (rms) beam emittance and $\gamma=\left(1-\beta^{2}\right)^{-1 / 2}$ is the beam relativistic factor with $\beta$ the beam velocity normalized by the speed of light. Here, the current pinching the beam is the total diode current and the emittance is primarily determined by the non-linear self-field interactions during transit across the A-K gap.

\section{Results and discussion}

Typical parameters for optimized diodes are $\gamma=14$, $\varepsilon=1.5 \mathrm{~cm} / \mathrm{rad}, I_{\mathrm{smp}}=150 \mathrm{kA}, r_{\mathrm{c}}=0.6 \mathrm{~cm}$, and $d=1.2 \mathrm{~cm}$. These configurations yield doses of $\approx 350 \mathrm{rad}$ (measured at one meter) with $6.5 \mathrm{MeV}$ endpoint photon energy (the dose is measured through filtration equivalent to $16 \mathrm{~mm} \mathrm{Al}$ in order to attenuate low energy photons). The X-ray source profile based on the line-spread-function from a similar shot to that shown in shot 336 is shown in Fig. 2b. A fitted function comprised of a double Gaussian profile is also shown. The full-width-half-maximum (FWHM) of the profile is $1.7 \mathrm{~mm}$, with dual axis views indicating a symmetric spot. These recent results provide a source with an $\mathrm{FOM} \approx 50 \mathrm{rad} / \mathrm{mm}^{2}$. The dose agrees reasonably well with the predictions of Hinshelwood et al. [3], scaling nearly as dose $(\operatorname{rads} / \mathrm{mC}) \approx$ $0.86 \mathrm{~V}^{2.2}$ (the dose is normalized by the total electron charge deposited and we assume $10 \%$ ion current).

These optimized diodes usually have the geometric $\mathrm{A}-\mathrm{K}$ gap $d \approx 2 F_{\mathrm{smp}}$, whereas the measured X-ray spatial distribution (which is a direct image of the e-beam) supports the theoretically assumed minimum radius $a_{\text {min }}$. According to Eq. $(3), F_{\mathrm{smp}} \approx 0.7 \mathrm{~cm}$ and $a_{\min } \approx 0.14 \mathrm{~cm}$ for shot 336. Thus there is an inconsistency in the theoretical prediction of the focal length (Eq. (3)) and the operating impedance of the diode based on Eq. (2), unless one assumes that the actual $\mathrm{A}-\mathrm{K}$ gap (defined by the potential surfaces) is smaller than $d$ and that $\alpha$ is also correspondingly smaller.

Assuming that the actual $\mathrm{A}-\mathrm{K}$ gap is equal to $F_{\text {smp }}$, then substituting $F_{\text {smp }}$ from Eq. (3) for $d$ in Eq. (2) yields

$$
I_{\mathrm{smp}} \approx \frac{\alpha^{2}}{\pi} I_{\mathrm{A}}, \quad F_{\mathrm{smp}} \approx \frac{r_{\mathrm{c}}}{2} \frac{\pi}{\alpha}
$$

and for the above parameters $\left(r_{\mathrm{c}}=0.6 \mathrm{~cm}, \gamma=14\right.$, $\left.I_{\mathrm{smp}}=150 \mathrm{kA}, I_{\mathrm{A}}=232 \mathrm{kA}\right)$, one calculates $\alpha \approx 1.4$, which is in close agreement to large aspect ratio self-pinch diodes. Gated optical light images as well as hybrid particle-in-cell (PIC) simulations suggest that the impedance is probably due to plasma expansion from both the anode and cathode. The inference of a large 
$\alpha$ factor if one assumes the geometric gap $d$ in the calculation of $I_{\mathrm{smp}}$ and the effects associated with plasma closure suggest that more accurate models of the diode impedance need to consider self-consistent ion space charge profiles and need to be 2-dimensional since aspect ratios are of order 1 . In hybrid fluid/kinetic simulations it is observed that both the effective gap and the effective cathode radius $r_{\mathrm{c}}$ (defined as the radius where space-charge limited emission occurs) decrease simultaneously. This allows the diode impedance to be more stable than expected at these high power densities and is most likely allowing for the nearly $50 \mathrm{~ns}$ FWHM radiation pulse widths to be achieved.

\section{Acknowledgments}

Sandia is a multiprogram laboratory operated by Sandia Corporation, a Lockheed Martin Company, for the
United States Department of Energy's National Nuclear Security Administration under contract DE-AC0494AL85000.

\section{References}

[1] I.D. Smith, et al., in: Proc. 12th IEEE Int. Pulsed Power Conf., Monterey (CA, USA) 1999, p. 403.

[2] S.B. Swanekamp, G. Cooperstein, J.W. Schumer et al., IEEE Trans. Plasma Sci. 32, 2004 (2004).

[3] D.D. Hinshelwood, R.J. Allen, R. Commisso et al., IEEE Trans. Plasma Sci. 35, 565 (2007).

[4] G. Cooperstein et al., Phys. Plasma 8, 4618 (2001).

[5] B.V. Oliver, P.F. Ottinger, T.C. Genoni et al., Phys. Plasma 11, 3976 (2004). 